\title{
Thermal properties of clay-containing nanocomposite films
}

\author{
Hirotaka MAEDA ${ }^{\dagger}$, Tomoya SATO*, Matthew William ENGLAND*, \\ Atsushi HOZUMI* and Toshihiro KASUGA \\ Department of Life Science and Applied Chemistry, Nagoya Institute of Technology, \\ Gokiso-cho, Showa-ku, Nagoya 466-8555, Japan \\ *National Institute of Advanced Industrial Science and Technology (AIST), \\ 2266-98 Anagahora, Shimo-shidami, Nagoya 463-8560, Japan
}

\begin{abstract}
Thermal properties of superhydrophilic nanocomposite films prepared from polyvinylpyrrolidone and aminopropylfunctionalized clay were studied. The films were treated at various relative humidities to absorb water. They showed excellent water-absorption properties and their capacity (amount of water absorbed from the air) was found to be markedly influenced by the relative humidity. Absorbed water into the films led to enhancement of their thermal diffusivity. Due to the large amount of absorbed water by the films after the treatment at $\mathbf{9 8 \%}$, their thermal conductive properties were considerably improved. (c)2017 The Ceramic Society of Japan. All rights reserved.
\end{abstract}

Key-words : Nanocomposite film, Water absorption, Thermal property, Water vapor treatment

[Received July 13, 2017; Accepted September 27, 2017]

\section{Introduction}

Engineered thermal materials, such as carbon nanotube and phase change materials, have been widely used in various advanced applications for the purpose of thermal management. ${ }^{1), 2)}$ Thermal conduction is one of the fundamental physical parameters for thermal properties. For solid materials with the exception of metals, thermal conductivity is proportional to velocity of sound wave in a material, heat capacity, and phonon mean free path. ${ }^{3)}$ There is currently a demand for new materials possessing both higher heat capacity and longer phonon mean free paths, to improve their thermal conduction.

It is well known that water possesses a high specific heat compared to other liquids, which is closely related to its excellent heat capacity. We have previously reported that the infusion of water vapor into the nanopores of diatom frustules greatly improved their thermal conduction. ${ }^{4)}$ Recently, some of the authors have also successfully prepared transparent superhydrophilic nanocomposite films, which were prepared from polyvinylpyrrolidone (PVP) and aminopropyl-functionalized clay (AMPclay), showing excellent anti-fogging and self-healing properties. The composite films also exhibited excellent water absorption properties, as they were able to absorb an average of $166.7 \mathrm{wt} \%$ water from humid air within $24 \mathrm{~h}$. This great absorption ability is expected to improve the composite films' thermal conductive properties, which have not yet been examined. Thus, in this study we have particularly focused on the effects of relative humidity (RH) on both the composite films total absorption of water from the air, and their thermal conductive properties.

\section{Experimental procedures}

The superhydrophilic nanocomposite thin films, which primarily consisted of PVP and AMP-clay, were prepared using a slightly modified version of a procedure from our previous study. ${ }^{5), 6)}$ First, AMP-clay was prepared based on a sol-gel method. Briefly,

\rceil Corresponding author: H. Maeda; E-mail: maeda.hirotaka@ nitech. ac.jp
$0.84 \mathrm{~g}$ (3.62 mmol) of $\mathrm{MgCl}_{2} \cdot 6 \mathrm{H}_{2} \mathrm{O}$ was dissolved in ethanol, after which $1.3 \mathrm{~mL}(5.85 \mathrm{mmol})$ of 3-aminopropyltriethoxysilane (APTES) was added to the solution, and stirred overnight at room temperature. The resulting AMP-clay was then centrifuged and washed with excess ethanol, and finally dried in a fume hood at room temperature overnight. PVP was dissolved in $0.5 \mathrm{~mL}$ of water at $6 \mathrm{wt} \%$, and was mixed with $40 \mu \mathrm{L}$ of $10 \%$ glutaraldehyde (GA) solution. $0.5 \mathrm{~mL}$ of a $1.5 \mathrm{wt} \%$ aqueous AMP-clay dispersion was then added and stirred into the PVP/GA mixture ( $3 \mathrm{wt} \%$ PVP, $0.75 \mathrm{wt} \%$ AMP and $4.24 \times 10^{-3} \mathrm{wt} \%$ GA), and then immediately spin-coated onto the AMP-functionalized $\mathrm{Si}$ substrate (modified by a chemical vapor deposition of APTES at a temperature of $100^{\circ} \mathrm{C}$ for $1 \mathrm{~h}$ ). In order to increase coating thickness and stability, a second, identical PVP/AMP/GA mixture was prepared and deposited on top of the same sample around $5 \mathrm{~min}$ after the first deposition. The samples were then left to dry in a fume hood overnight.

Before starting water vapor treatments, all samples were dried at $60^{\circ} \mathrm{C}$ for $24 \mathrm{~h}$, and then were left in a box kept at 35,75 , and $98 \% \mathrm{RH}$, which was controlled using $\mathrm{MgCl}_{2}-\mathrm{H}_{2} \mathrm{O}$ mixtures, $\mathrm{NaCl}-\mathrm{H}_{2} \mathrm{O}$ mixtures, and $\mathrm{H}_{2} \mathrm{O}$, respectively, for $24 \mathrm{~h}$. In order to evaluate the ambient water-absorption capacity of our samples, changes in the weight of the samples before and after water vapor treatments were measured. The as-synthesized and vapor-treated samples were also characterized by X-ray diffraction (XRD) and Fourier transform infrared spectroscopy (FT-IR). To evaluate the thermal conductive properties of the samples, the films' surfaces were placed in direct contact with a heater kept in the range of 25 to $60^{\circ} \mathrm{C}$. Changes in surface temperature at the opposite side of the samples (before and after water vapor treatment) were monitored using a thermocouple. The thermal diffusivity of the samples, which is one of thermal properties, was determined by a laser flash method. ${ }^{7)}$ Each measurement was carried out at least three times.

\section{Results and discussion}

Weights of the samples increased to $0.7 \pm 0.2,0.9 \pm 0.1$, and $1.3 \pm 0.3 \mathrm{mg}$ after water vapor treatments at 33,75 , and $98 \%$, 


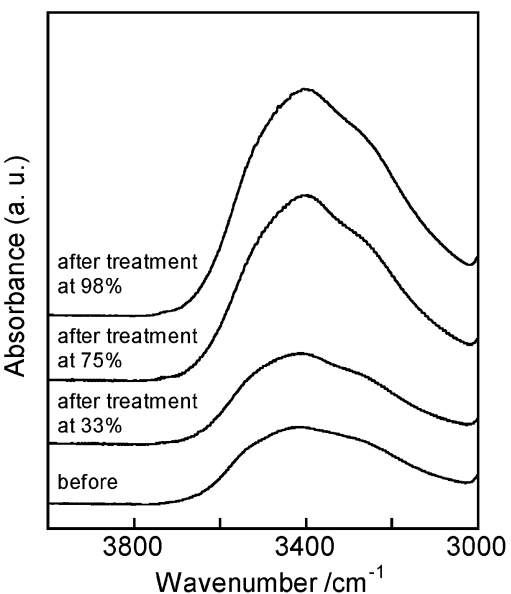

Fig. 1. FT-IR spectra of samples before and after water vapor treatments at different relative humidity.

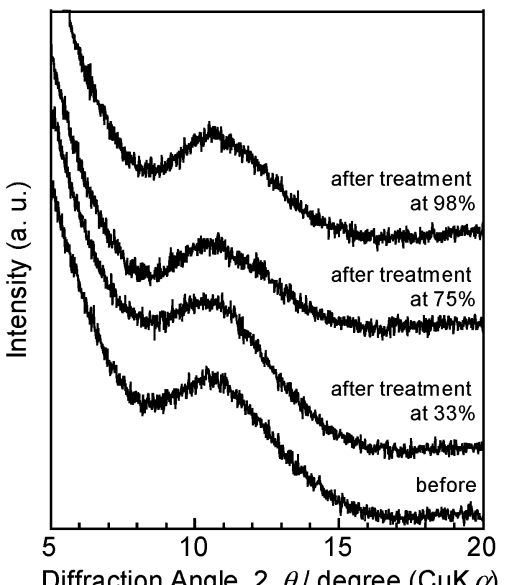

Fig. 2. XRD patterns of samples before and after water vapor treatments at different relative humidity.

respectively. This clearly demonstrates that our composite films can absorb water by the water vapor treatments, and the final amount of water absorbed was found to be markedly dependent on the RH. Figure 1 shows FT-IR spectra of the samples with and without water vapor treatments at different RH. In the case of samples before and after water vapor treatment at $33 \% \mathrm{RH}$, broad absorption bands corresponding to the stretching vibrations of structural hydroxyl groups in the brucite sheets ${ }^{8)}$ and water molecules interacting with carbonyl group of $\mathrm{PVP}^{9)}$ can be seen at around $3450 \mathrm{~cm}^{-1}$, and between 3300 and $3500 \mathrm{~cm}^{-1}$, respectively. No marked differences were observed between these two spectra. However, these absorption bands markedly increased with the RH of more than $75 \%$, and showed a strong correlation with the results of weight changes, as mentioned previously. XRD patterns of the identical samples used in FT-IR measurements were also shown in Fig. 2. A broad peak due to the lamellar stacked structure of AMP-clay ${ }^{10), 11)}$ was observed at around $10.5^{\circ}$ $(\mathrm{d}=0.86 \mathrm{~nm})$ in the sample before and after water vapor treatments. Different to the results shown in Fig. 1, the XRD pattern remained unchanged even after the treatment. From these result, it is implied that a higher water absorption into the films has no influence on the lamellar stacked structure.

The thermal diffusivity of the samples before and after water vapor treatments at $98 \%$ was determined to be $2.0 \pm 0.3 \times 10^{-5}$

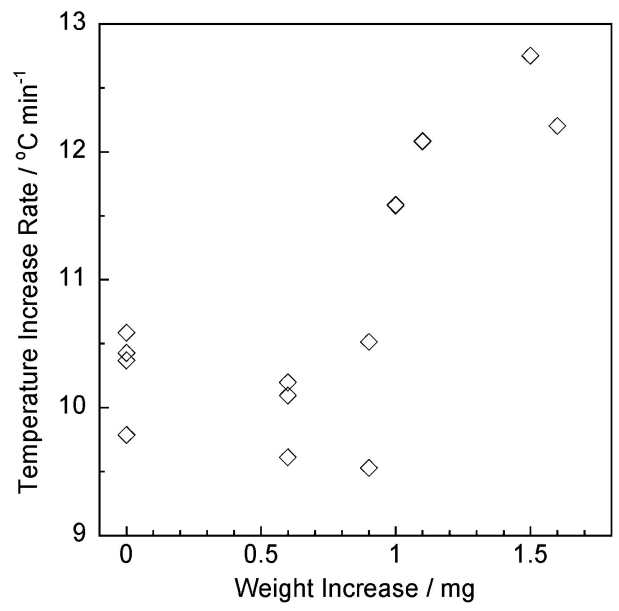

Fig. 3. Relationship between temperature increase rate of samples and weight increase after the water vapor treatments. Point indicated by zero represents the untreated sample.

and $2.3 \pm 0.2 \times 10^{-5} \mathrm{~m}^{2} / \mathrm{s}$, respectively. This increase in thermal diffusivity must be related to the water absorbed within the samples. In the case of nanocomposites, the addition of high thermal conductive materials, for example, graphene and carbon nanotube and etc., into matrix has been demonstrated to enhance the thermal conductivity. ${ }^{12)-14)}$ To our knowledge, no utilizing absorbed water for improving thermal conductive properties exist in the literature. The final temperatures of all samples with and without water vapor treatments were almost identical (approximately $59^{\circ} \mathrm{C}$ ) when the heater temperature was increased to $60^{\circ} \mathrm{C}$. The increase rate of the initial sample was determined to be $10.3 \pm 0.3^{\circ} \mathrm{C} / \mathrm{min}$, which remained nearly constant after treatment at $33 \% \mathrm{RH}$. Judging from all data, the increase in the samples' temperatureincrease rate seems to be closely related to the weight increase more than $1 \mathrm{mg}$ due to the water absorption, as shown in Fig. 3. We speculate the improvement of thermal conductive properties after water vapor treatments at higher RHs, as follows. The crystallinity of matrix in nanocomposite has been reported to influence their thermal conductivity. ${ }^{15), 16)}$ The full-width half maximum of the peak for AMP-clay in the XRD patterns (Fig. 2) was considered almost constant regardless of the water vapor treatment. It has been reported that a thermal conductivity is reduced by phonon scattering at the boundaries. ${ }^{17)}$ The thermal conductive percolation behavior plays an important factor in the great enhancement of the thermal conductivity. ${ }^{18)}$ The water vapor treatment causes an increase in the amount of water absorption in the films. The larger amount of absorbed water after water vapor treatments at higher RHs is proposed to percolate through the film. As a result, this would act as thermal conductive pathway in the film, leading to enhancement of thermal conductive properties.

\section{Conclusions}

We have found that our PVP and AMP-clay composite films possess an excellent capacity for absorption of ambient water. The level of water absorption was markedly influenced by $\mathrm{RH}$ during the vapor-exposure treatments. In particular, under higher $\mathrm{RH}$ conditions of more than $75 \%$, marked increases in water absorption and changes in the film structures were confirmed by measuring weight difference, FT-IR and XRD. Thanks to the large amount of absorbed water in the films, their thermal conductive properties were considerably improved. 
Acknowledgement This work was supported by JSPS KAKENHI Grant Number JP24120005 in Scientific Research on Innovative Areas "Innovative Materials Engineering Based on Biological Diversity".

\section{References}

1) A. Yu, P. Ramesh, X. Sun, E. Bekyaravo, M. E. Itkis and R. C. Haddon, Adv. Mater., 20, 4740-4744 (2008).

2) T. Matsunaga, N. Yamada, R. Kojima, S. Shamoto, M. Sato, H. Tanida, T. Uruga, S. Kohara, M. Takata, P. Zalden, G. Bruns, I. Sergueev, H. C. Wille, R. P. Hermann and M. Wuttig, $A d v$. Funct. Mater, 21, 2232-2239 (2011).

3) C. Kittele, Phys. Rev., 75, 972-974 (1949).

4) H. Maeda, M. Matsumoto, Y. Maeda, Y. Egashira, T. Tanaka and T. Kasuga, J. Appl. Phycol., in press.

5) M. W. England, C. Urata, G. J. Dunderdale and A. Hozumi, ACS Appl. Mater. Interfaces, 8, 4318-4322 (2016).

6) M. W. England, T. Sato, C. Urata, L. Wang and A. Hozumi, J. Colloid Interf. Sci., 505, 566-576 (2017).

7) W. J. Parker, R. J. Jenkins, C. P. Butler and G. L. Abbott, J. Appl. Phys., 32, 1679-1684 (1961).
8) H.-Y. Zeng, J.-Z. Du, S. Xu, M.-C. Liao, X.-J. Liu, H.-Z Dduan and C.-R. Chen, RSC Adv., 5, 64814-64820 (2015).

9) L.-S. Wan, X.-J. Huang and Z.-K. Xu, J. Phys. Chem. B, 111, 922-928 (2007).

10) N. T. Whilton, S. L. Burkett and S. Mann, J. Mater. Chem., 8, 1927-1932 (1998).

11) J. E. Matrtin, A. J. Patil, M. F. Butler and S. Mann, Adv. Funct. Mater., 21, 674-681 (2011).

12) V. Datsyuk, S. Trotsenko and S. Reich, Carbon, 52, 605-608 (2013).

13) S. Harish, D. Orejon, Y. Takata and M. Kohno, Appl. Therm. Eng., 80, 205-211 (2015).

14) X. Huang, C. Zhi, P. Jiang, D. Golberg, Y. Bando and T. Tanaka, Adv. Funct. Mater., 23, 1824-1831 (2013).

15) R. Haggenmuller, C. Guthy, J. R. Lukes, J. E. Fischer and K. I. Winey, Macromolecules, 40, 2417-2421 (2007).

16) J. Gu, N. Li, L. Tian, Z. Lv and Q. Zhang, RSC Adv., 5, 3633436339 (2015).

17) D. L. Nika, A. I. Cocemasov, D. V. Crismari and A. A. Balandin, Appl. Phys. Lett., 102, 213109 (2013).

18) N. Shenogina, S. Shenogin, L. Xue and P. Keblinski, Appl. Phys. Lett., 87, 133106 (2005). 